\title{
Epidemiology of human brucellosis in Nahavand county, Hamadan Province, western Iran: an 8-year (2010-2017) registry-based analysis
}

\author{
Salman Khazaei ${ }^{1 \oplus}$, Manoochehr Solgi ${ }^{1 \oplus}$, Shahram Goodarzi ${ }^{\circledR \oplus}$, Leila Khazaei ${ }^{3 \oplus}$, Iraj Salehi ${ }^{4 \oplus}$, \\ Ensiyeh Jenabi ${ }^{5, *} \odot$
}

\section{Abstract}

Background: Brucellosis is an infectious zoonosis, which greatly afflicts human health and animal productivity.

Objectives: To describe the trends and epidemiological characteristics of human brucellosis in Nahavand county, Hamadan Province, western Iran over 8 years (2010-2017).

Methods: In this registry-based longitudinal study, we analyzed all reported cases of human brucellosis, including 1,130 patients from 2010 to 2017. A checklist including demographic and clinical characteristics of patients with reported cases of brucellosis had been used to gather data. We calculated annual percent changes (APCs) and average annual percent changes (AAPCs) using Joinpoint software to determine the trend of brucellosis using a segmented regression model.

Results: Among the patients, $60.9 \%$ were male and $87.3 \%$ lived in rural areas. The mean age of the patients was $35.9 \pm 18.34$ years. Compared with male patients, female patients were more affected when they were elderly $(15.6 \%$ men vs. $24.2 \%$ women for those aged $\geq 55$ years $)(P=0.001)$. Of patients with brucellosis, $65.2 \%$ had consumed unpasteurized dairy products and $82.3 \%$ had a history of contact with animals. The incidence of human brucellosis in Nahavand county increased between 2010 and 2014, then decreased in 2015, and thereafter remained steady. Per 100,000 population, the AAPC pertaining to the incidence was 17.4 in the male population (95\% CI: 4.4, 31.9), 13.8 in the female population $(95 \%$ CI: $0.2,29.3)$, and 16.1 in rural dwellers $(95 \%$ CI: $2.2,31.8)$ indicating an increasing trend from 2010 to $2017(P<0.05)$.

Conclusion: The incidence of brucellosis in the western part of Iran is high and remains a challenging health problem. In the present study, age, job, sex, and seasonal changes are important risk factors for human brucellosis.

Keywords: brucellosis; epidemiology; health surveys; Iran; public health surveillance

\footnotetext{
*Correspondence to: Ensiyeh Jenabi, Pediatric Developmental Disorders Research Center, Hamadan University of Medical Sciences, Shariati Street, Hamadan 65178-38695, Iran, e-mail: e.jenabi@umsha.ac.ir

'Department of Epidemiology, School of Public Health, Hamadan University of Medical Sciences, Hamadan 65178-38695, Iran 2Department of Public Health, School of Public Health, Hamadan University of Medical Sciences, Hamadan 65178-38695, Iran ${ }^{3}$ Department of Epidemiology, School of Health and Safety, Shahid Beheshti University of Medical Sciences, Tehran 19839-63113, Iran ${ }^{4}$ Department of Anesthesiology, School of Allied Medical Sciences, Hamadan University of Medical Sciences, Hamadan 65178-38695, Iran ${ }^{5}$ Autism Spectrum Disorders Research Center, Hamadan University of Medical Sciences, Hamadan 65178-38695, Iran
} 
Brucellosis, also known as "undulant fever," "Mediterranean fever," "Malta fever," "Gibraltar fever," "thousand face disease," and "raging fever" is caused by Brucella species. Brucellosis is a highly infectious zoonosis, which greatly afflicts human health and animal productivity. Brucella spp. are gram-negative aerobic bacteria and are destroyed by boiling or pasteurization [1]. Brucellosis can be transmitted from animal reservoirs to humans by the consumption of raw animal products, such as unpasteurized milk or cheese, or by direct contact with infected animals [2]. Human brucellosis is considered an occupational risk, which affects people who work with infected animals or their tissues including farmers, veterinarians, abattoir workers, and livestock breeders $[1,3]$. The symptoms of infection in human brucellosis are nonspecific, but the most common manifestations are fever, fatigue, backache, headache, and arthralgia [4, 5]. Some conditions, such as poor farm hygiene, unrestricted trade, movement of animals from one place to another or between countries, and weakness in livestock surveillance, can play a major role in the spread of infection [6].

An accurate estimation of the incidence of human brucellosis is difficult due to underestimated reports and misdiagnosis. However, the incidence of brucellosis is higher in rural areas than in urban areas. Human brucellosis has higher proportions of treatment failure and results in fundamental residual disability of infected patients and the incidence of high recurrence [5].

Human brucellosis is one of the commonest zoonotic diseases and $>500,000$ new cases were reported annually worldwide [7]. While brucellosis is globally widespread and more common in less developed regions, such as Asia, Africa, Central and South America, and the Mediterranean $[8,9]$, it is a rare phenomenon in advanced countries because of eradication programs [10]. For example, in France, according to human brucellosis surveillance, a total of 250 brucellosis cases were notified ( 0.3 cases per million) from 2004 to 2013 [11].

Iran is identified as an endemic area for human brucellosis where it remains a serious public health issue [12]. According to the results of a meta-analysis conducted in Iran by Mirnejad et al. in 2017, the incidence of brucellosis is $0.001 \%$ annually and ranged from 7.0 per 100,000 to 276.41 per 100,000 population in Qom and Kermanshah provinces, respectively. The highest incidences of brucellosis occur in the west and northwest regions of Iran. Overall, the incidence of the disease in Iran is high [13].

Ghadimi-Moghadam et al. in their study regarding the effect of ecological factors on the distribution of brucellosis in Kohgiluyeh and Boyer-Ahmad Province found that the densities of human, sheep, goat, and cow population were positively associated with the occurrence of the disease. Geo-climatic factors of elevation, slope, and rainfall were negatively associated with the disease [14]. Location and time are the 2 important factors affecting the distribution of brucellosis.

The present study aimed to analyze the trend and epidemiological characteristics of human brucellosis in Nahavand county, Hamadan Province, western Iran over 8 years (2010-2017). The findings of this study may give a better understanding of the magnitude of the disease and guide policymakers in the design of prevention and control strategies.

\section{Methods}

\section{Study area}

This study was conducted on Nahavand county of which Nahavand City is the capital. The county is located in southern Hamadan Province, in western Iran. Based on the 2016 census, its population was 178,787 , of which 85,333 people lived in rural areas and 91,978 lived in urban centers. Nahavand lies in an elevated region; its climate is a local steppe climate with a light annual average rainfall of just $379 \mathrm{~mm}$, with warm summers and relatively cold winters. Due to appropriate vegetation, this district is known as a hub of animal husbandry.

\section{Study design}

The present study is a registry-based longitudinal study of 1,130 reported cases of human brucellosis from 2010 to 2017.

\section{Ethics}

The study protocol was approved by the Ethics Committee of the Hamadan University of Medical Sciences (reference No. IR.UMSHA.REC.1398.519).

\section{Data sources}

A checklist including demographic and clinical characteristics of reported cases of brucellosis from private and public laboratories to the Nahavand Health Center was used to curate data. This checklist included sex, age group, location (urban/rural), city, time of diagnosis (year), disease type (new vs. recurrent case), family history of the disease, animal contact history, and history of unpasteurized dairy consumption. In Iran, according to the brucellosis national surveillance system, patient assessment forms for reported cases of brucellosis are completed 
by health experts in Health Centers through interviews after patient consent as a routine task.

\section{Brucellosis case definition}

According to Iranian national guidelines, a case of brucellosis is defined as the presence of confirmed clinical diagnosis based on compatible signs and symptoms, standard tube agglutination test $\geq 1: 80$ and 2-mercaptoethanol agglutination $\geq 40$ [1]. Only confirmed cases are recorded in the Iranian national registry program for brucellosis. In the present study, we excluded only non-native patients from the final analysis (8 cases).

\section{Statistical analysis}

We used descriptive statistics including frequency, percentage, and bar and radar charts (MS Excel) to describe the study variables.

Segmented analysis was used because of the nonconstant trend for the incidence of brucellosis over the study time. This model assumes that the change in the incidence is constant over each time segment called change points but varies between different time segments [15]. Joinpoint regression is a valuable tool for making inferences about changes in trends over time [16]. The tool, also known as change-point regression or segmented regression, assumes that data can be divided into subsets - each with its unique linear trend. In our present study, the incidence from 2010 to about 2013 increased and after that it decreased. Therefore, due to this change point in the trend of the disease using this model is suitable. In the present study, of the trend, the annual percent change (APC) and the average annual percent change (AAPC) of brucellosis were considered as independent variables, whereas, the incidence of brucellosis by sex and location (per 100,000 population) during 2010-2017 was considered dependent. The APC is one way to measure the trends of the disease over time, and the AAPC is a summary of the trend and determines the years to determine the summary statistics of the trend.

Data were analyzed using the Joinpoint Regression Program (version 4.7.0.0, Feb 2020; Statistical Methodology and Applications Branch, Surveillance Research Program, National Cancer Institute). Differences with $P<0.05$ were considered significant.

\section{Results}

\section{Analysis of baseline characteristics}

During the 8 years covered by the study (2010-2017), a total of 1,130 cases of human brucellosis were registered in Nahavand county. The vast majority of the patients $(90.4 \%)$ had new cases. Table 1 shows the sex and location of study participants according to their age group. Among all the patients, $60.9 \%$ were male and $87.3 \%$ lived in rural areas. The mean age of the patients was $35.9 \pm 18.34$ years and 735 patients $(38.5 \%)$ were 24-44 years old, while $140(12.4 \%)$ were $<15$ years old. Compared with male patients, female patients were more affected when they were elderly $(15.6 \%$ men vs. $24.2 \%$ women for those aged $\geq 55$ years $)(P<0.001)$. Of patients with brucellosis, $75.9 \%$ reported no history of brucellosis in their family, while $65.2 \%$ had consumed unpasteurized dairy products, and $82.3 \%$ had a history of contact with animals (Table 1).

According to the seasonality of reports, the greatest proportions of the cases occurred in summer (June [13.5\%] and July [13.4\%]), while the lowest proportion occurred in winter (November [5.3\%] and January [5.7\%]) (Figure 1).

Human brucellosis in Nahavand was most common among farmers $(56.0 \%)$ and female housekeepers (18.7\%) (Figure 2).

Table 1. Sex and rural-urban divide of reported cases of human brucellosis by age group, in Nahavand county (2010-2017)

\begin{tabular}{|c|c|c|c|c|c|c|c|}
\hline \multicolumn{2}{|c|}{$\begin{array}{l}\text { Age group } \\
\text { Frequency (\%) }\end{array}$} & \multirow{2}{*}{$\begin{array}{c}\begin{array}{c}\text { 0-14 y } \\
\text { n (\%) }\end{array} \\
98(14.2)\end{array}$} & \multirow{2}{*}{$\begin{array}{c}\begin{array}{c}15-24 \\
\text { n }\end{array} \\
123(17.9)\end{array}$} & \multirow{2}{*}{$\begin{array}{c}\begin{array}{c}25-44 \\
\text { n }\end{array} \\
274(39.8)\end{array}$} & \multirow{2}{*}{$\begin{array}{c}\begin{array}{c}45-54 \\
\text { y }\end{array} \\
86(12.5)\end{array}$} & \multirow{2}{*}{$\begin{array}{c}\begin{array}{c}\mathbf{5 5}+\mathbf{y} \\
\mathbf{n}(\%)\end{array} \\
107(15.6)\end{array}$} & \multirow{2}{*}{$\begin{array}{c}P \\
<0.001 *\end{array}$} \\
\hline Sex & $\begin{array}{l}\text { Male } \\
668(60.9 \%)\end{array}$ & & & & & & \\
\hline & $\begin{array}{l}\text { Female } \\
442(39.1 \%)\end{array}$ & $42(9.5)$ & 64 (14.5) & $161(36.4)$ & 68 (15.4) & $107(24.2)$ & \\
\hline \multirow[t]{2}{*}{ Location } & $\begin{array}{l}\text { Rural } \\
986 \text { (87.3) }\end{array}$ & $125(12.7)$ & $163(16.5)$ & $376(38.1)$ & 135 (13.7) & $187(19.0)$ & 0.94 \\
\hline & $\begin{array}{l}\text { Urban } \\
144 \text { (12.7\%) }\end{array}$ & $15(10.4)$ & 24 (16.7) & $59(41.0)$ & $19(13.2)$ & 27 (18.8) & \\
\hline Total & & $140(12.4)$ & $187(16.6)$ & $435(38.5)$ & 154 (13.6) & 214 (18.9) & \\
\hline
\end{tabular}

${ }^{*} P<0.05$ (highlighted in bold). 


\section{Time trend analysis}

The incidence of human brucellosis in Nahavand increased between 2010 and 2014 (27.5, 51.7, 69.1, 109.0, and 121.4 per 100,000 population for 2010, 2011, 2012, 2013, and 2014, respectively), then decreased in 2015, thereafter remained steady $(84.3,85.4$, and 86.5 per 100,000 population for 2015 , 2016, and 2017, respectively).

There was one point (year) of change for all groups (Table 2). The AAPC for incidence per 100,000 in the male population was $17.4(95 \% \mathrm{CI} 4.4,31.9)$, in the female

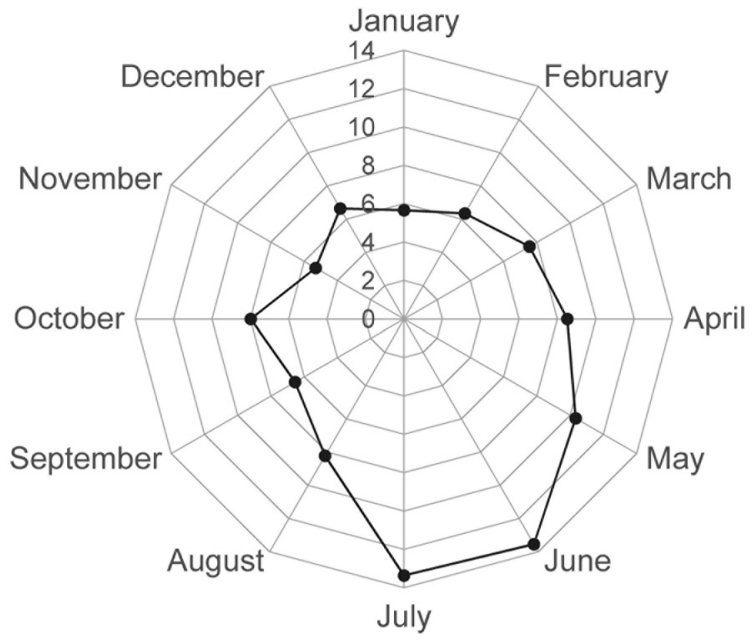

Figure 1. Radar plot showing average morbidity (\%) and month of 1,130 reported cases of human brucellosis in Nahavand county, western Iran (2010-2017). population was 13.8 (95\% CI $0.2,29.3)$, and in the rural population was 16.1 (95\% CI 2.2, 31.8), each statistically significant, with a positive sign indicating an increasing trend during the study period. This means the 1-year increase had resulted in a $17.4 \%$ increase in the incidence in the male population, $13.8 \%$ in the female population, and $16.1 \%$ in rural dwellers. Although, according to the second segments and based on APC, we observed a decreasing trend for all the groups of interest, which varied from -4.0 (for incidence in the female population) to -15.3 (incidence rate in urban areas). The results of APC for human brucellosis incidence by sex and urban-rural divide are shown graphically in Figure 3.

\section{Discussion}

The findings of this study show that brucellosis is a major health problem in Nahavand county. In this county, the incidence of human brucellosis was much higher than in many other counties in Iran. The occurrence of brucellosis in Nahavand has not been regular and increased between 2010 and 2014 , then decreased in 2015, and thereafter remained steady.

In the present study, men aged 25-44 years and residents of rural regions were found at the highest risk of acquiring this disease. As consistent with our findings, Nematollahi et al. had made similar findings in Hamadan province. In their study, $>60 \%$ of the patients were male; $35 \%$ of patients were in an economically active age group ( $25-44$ years), and $>80 \%$ were rural residents [17]. It should be noted that Nahavand is one of the counties of Hamadan Province with a high prevalence

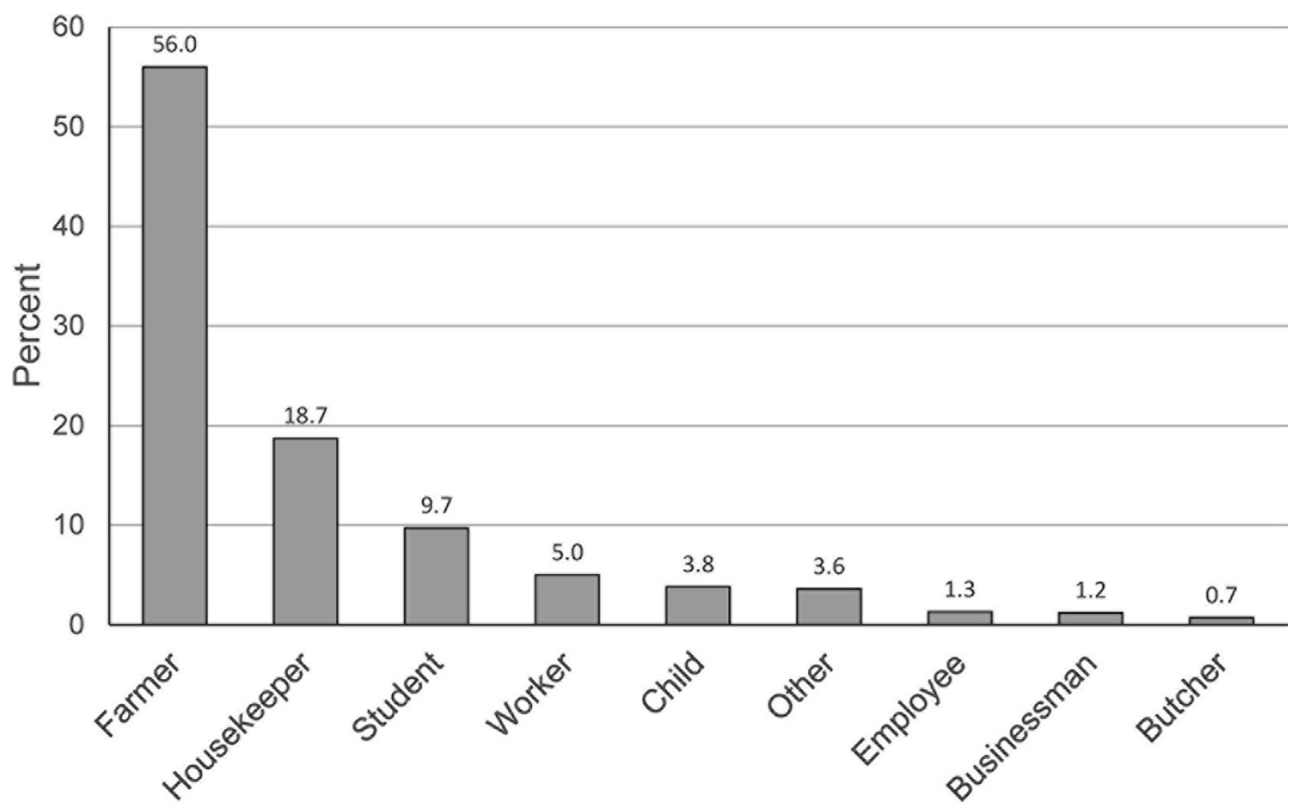

Figure 2. Occupation of 1,130 patients with reported cases of human brucellosis in Nahavand county, western Iran (2010-2017). 
Table 2. Year of change point, APC, and AAPC for brucellosis incidence in Nahavand, 2010-2017

\begin{tabular}{|c|c|c|c|c|c|c|}
\hline Group & No. of change points (year) & Years & $\begin{array}{c}\text { APC } \\
\text { Mean }(95 \% \mathrm{Cl})\end{array}$ & $P$ & $\begin{array}{c}\text { AAPC } \\
\text { Mean }(95 \% \mathrm{Cl})\end{array}$ & $P$ \\
\hline \multirow[t]{2}{*}{ Male population } & $1(2013)$ & $2010-2013$ & $68.5 *(20.1,136.4)$ & $0.016^{*}$ & $17.4(4.4,31.9)$ & $0.007^{*}$ \\
\hline & & 2013-2017 & $-10.5(-27.8,10.8)$ & 0.20 & & \\
\hline \multirow[t]{2}{*}{ Female population } & $1(2013)$ & 2010-2013 & $42.8(-1.3,106.5)$ & 0.055 & $13.8(0.2,29.3)$ & $0.047^{*}$ \\
\hline & & 2013-2017 & $-4.0(-24,21.3)$ & 0.62 & & \\
\hline \multirow[t]{2}{*}{ Urban population } & $1(2014)$ & 2010-2014 & $36.2(-2.9,91.1)$ & 0.062 & $11.1(-7.6,33.7)$ & 0.26 \\
\hline & & 2014-2017 & $-15.3(-50.4,44.7)$ & 0.40 & & \\
\hline \multirow[t]{2}{*}{ Rural population } & 1 (2013) & 2010-2013 & $59.2(10.3,129.9)$ & $0.028 *$ & $16.1(2.2,31.8)$ & $0.02 *$ \\
\hline & & 2013-2017 & $-8.4(-27.4,15.5)$ & 0.31 & & \\
\hline \multirow[t]{2}{*}{ Overall } & $1(2013)$ & 2010-2013 & $57.3(13.6,117.9)$ & $0.021^{*}$ & $15.8(3.5,29.6)$ & $0.01 *$ \\
\hline & & 2013-2017 & $-8.0(-25.1,13.1)$ & 0.23 & & \\
\hline
\end{tabular}

APC, annual percent change; AAPC, average annual percent change.

${ }^{*} P<0.05$ (highlighted in bold).
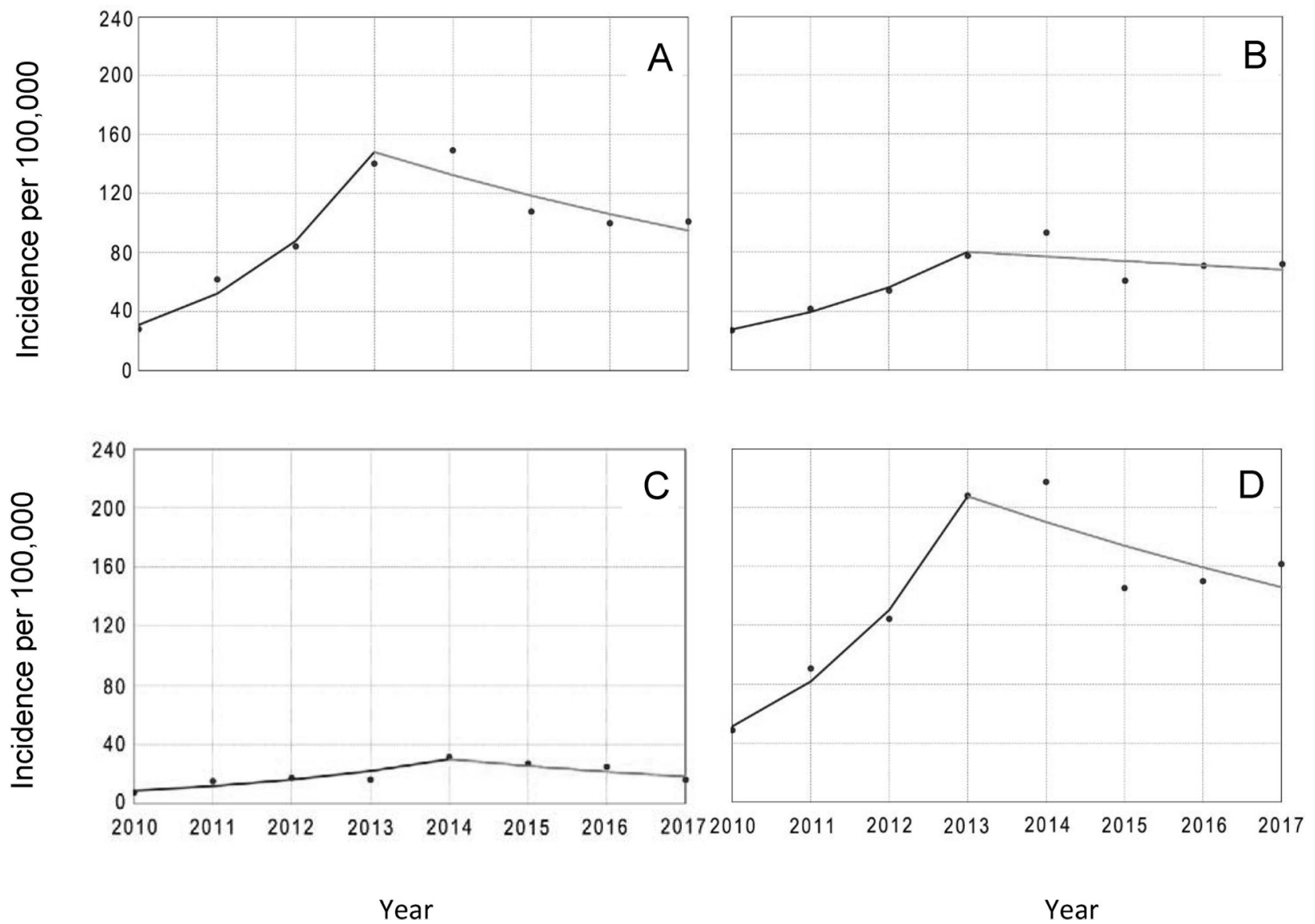

Figure 3. Trend and annual percent change (APC) for brucellosis incidence in Nahavand county, western Iran (2010-2017). Incidence is shown per 100,000 population (solid circles indicated observed values). (A) Male population, dark gray line 2010-2013 APC 68.5*, light gray line 2013-2017, APC -10.5; (B) female population, dark gray line 2010-2013 APC 42.8, light gray line -4; (C) urban population, dark gray line 2010-2014 APC 36.2, light gray line -15.3; (D) rural population, dark gray line 2010-2013 APC 59.2*, light gray line 2013-2017-8.4 (see Table 2). ${ }^{*} P<0.05$. Joinpoint Regression Program (version 4.7.0.0, Feb 2020; Statistical Methodology and Applications Branch, Surveillance Research Program, National Cancer Institute). 
of brucellosis, and parts of data used in the present study were also applied in the study by Nematollahi et al. In the present study, we used more updated data and assessed the trend of the disease over time, whereas Nematollahi et al. focused only on the epidemiological characteristics of the disease in Hamadan Province.

In a study of the Duhok Governorate in Iraq's Kurdis$\tan$ Region, the highest rate of brucellosis was observed in those aged 21-30 years [18]. Aloufi et al. made similar findings in their study in Saudi Arabia [19]. Age is an important risk factor for brucellosis worldwide. This young (21-30 y) group is economically active in society, may be more likely to come into contact with infected animals, as they travel more than women, and have a greater probability of drinking unpasteurized milk during the spring and summer seasons. Direct contact with livestock and the finding of brucellosis as an occupational disease can explain the greater occurrence of brucellosis in rural areas. However, there are some conflicting results; for example, some studies have shown that female sex was a risk factor for brucellosis $[18,20,21]$. The level of involvement of women in the handling of milk and other dairy products can account for these apparent discrepancies.

In the present study, $12.4 \%$ of cases were in patients $<15$ years old. This suggests that in Nahavand county and other developing societies, brucellosis is not uncommon among children. Khazaei et al. made a similar finding in their study of Hamadan province [22]. They showed that in this endemic region of brucellosis, childhood brucellosis includes up to one-third of all cases of human brucellosis.

In the present study, the greatest proportions of the cases occurred in the summer and farmers and female housekeepers were more at risk. During summer, direct contact between ranchers and aborted animal fetuses and increased consumption of contaminated dairy products increase the incidence of brucellosis. This finding is in accordance with the results of a systematic review of human brucellosis in Iran by Moosazadeh et al., which showed that the incidence of brucellosis in Iran during spring and summer seasons was higher than in winter and autumn seasons when the incidence of the disease decreases [23]. Other studies in Iran, Saudi Arabia, and Greece have made similar findings [24-26]. The present study confirmed the findings of the study by Rostami et al. [27], and a study conducted in Saudi Arabia shows that the highest number of cases was from April to June [28].

Rostami et al. showed that mean brucellosis incidence of 1,698 patients from 30 provinces of Iran was 29.83 per 100,000 population [27]. Bokaie et al. reported an incidence of brucellosis of 37 per 100,000 population in eastern Iran
[29]. Kassiri et al. found that the incidence of brucellosis in western Iran was 59.31 per 100,000 population [24]. Thus, western Iran has a higher incidence of brucellosis than eastern Iran. Hamadan is a province with a very high incidence of brucellosis (31-41 per 100,000 population) and Nahavand county had an incidence of 86.52 per 100,000 population in 2017 and also being extremely high within the province. Therefore, it seems necessary the need to increase the health surveillance system, especially in Nahavand county. This can be achieved by training health workers in the public and private sectors.

Like us, Kansiime et al. [30] in their study conducted of the south-western region of Uganda found an increasing trend of brucellosis incidence between the years 2010 and 2012. They attributed this increasing trend to improved health-seeking behavior of patients due to an increase in their health education about brucellosis through local radio stations. Some factors can be attributed to the increasing trend in our study; for example, by promoting public health awareness of people through training provided by health centers and clinics, more people come forward to get tested. Given that the incidence of the disease in livestock is closely related to the incidence of human brucellosis, the status of vaccination of livestock in these years can determine the incidence of disease in the human population. However, we could not determine the vaccination status of livestock in these years in the county. Given that Nahavand has always been one of the counties with the highest incidence of disease, effective educational interventions have been conducted in recent years and vaccination of livestock in high-prevalence counties is a priority for veterinary medicine.

The present study has some limitations. First, due to the retrospective nature of our study, we could not access other patient information, such as education, economic status, following complications, or the exact nature of contact with animals. Second, we could not link our data to data from agriculture or veterinarians' reports for a better understanding of our findings. Third, we could not be assured that data with the same quality were gathered during the years investigated. Despite these limitations, the considerable sample size of the investigated patients, and the long period of the study can be considered as its strengths.

\section{Conclusions}

The incidence of brucellosis in the western parts of Iran is high, and this remains a challenging health problem. In the present study, age 24-44 years, occupation as a farmer, being male, and summer season are important risk factors for the 
acquisition of human brucellosis. There is a need to empower at-risk populations to control the risk. Determining the cause of the increasing trends of the disease in Nahavand warrants further investigation. It seems necessary to improve the health surveillance system, which can be achieved by training health workers in the public and private sectors.

Author contributions. SK and JE substantially contributed to the conception and design of this study. MS, SG, and LK curated the data and SG, LK, and IS analyzed and interpreted it. SK, IS, and JE drafted the original manuscript and all authors critically revised it. All authors approved the final version submitted for publication and take responsibility for the statements made in the published article.

Acknowledgment. The authors thank the Vice-chancellor of Research and Technology, Hamadan University of Medical Sciences for supporting the study financially (research id: 9807165318).

Conflict of interest statement. The authors have each completed and submitted an International Committee of Medical Journal Editors Uniform Disclosure Form for Potential Conflicts of Interest. None of the authors has declared any potential conflict of interest concerning the present work.

Data sharing statement. Data generated or analyzed during the present study are included in this published article. Data that support the findings of this study are also available in figshare, with the identifier https://doi.org/10.6084/ $\mathrm{m} 9$.figshare/12623600; and all data are available from the corresponding author on reasonable request after deidentification of data from any person whose data was included in the study.

\section{References}

[1] Seleem MN, Boyle SM, Sriranganathan M. Brucellosis: a re-emerging zoonosis. Vet Microbiol. 2010; 140:392-8.

[2] Godfroid J, Cloeckaert A, Liautard J-P, Kohler S, Fretin D, Walravens K, et al. From the discovery of the Malta fever's agent to the discovery of a marine mammal reservoir, brucellosis has continuously been a re-emerging zoonosis. Vet Res. 2005; 36:313-26.

[3] Lytras T, Danis K, Dounias G. Incidence patterns and occupational risk factors of human brucellosis in Greece, 2004-2015. Int J Occup Environ Med. 2016; 7:221-26.

[4] Golshani M, Buozari S. A review of brucellosis in Iran: epidemiology, risk factors, diagnosis, control, and prevention. Iranian Biomed J. 2017; 21:349-59.

[5] Dean AS, Crump L, Greter H, Hattendorf J, Schelling E, Zinsstag J. Clinical manifestations of human brucellosis: a systematic review and meta-analysis. PLoS Negl Trop Dis. 2012; 6:e1929. doi: 10.1371/ journal.pntd.0001929

[6] Kant N, Kulshreshtha P, Singh R, Mal A, Kumar S, Tehlan M, et al. Prevalence of brucellosis and awareness of its spread. DU J Undergrad Res Innov. 2015; 4:43-9.

[7] Godfroid J. Brucellosis in livestock and wildlife: zoonotic diseases without pandemic potential in need of innovative one health approaches. Arch Public Health. 2017; 75:34. doi: 10.1186/s13690-0170207-7

[8] Ducrotoy M, Bertu WJ, Matope G, Cadmus S, Conde-Álvarez R, Gusi AM, et al. Brucellosis in sub-Saharan Africa: current challenges for management, diagnosis and control. Acta Trop. 2017; 165:179-93.

[9] Sohrabi A, Hajia M. Brucellosis: current situation in Iran and neighboring countries. Infect Disord Drug Targets. 2019. doi: 10.217 $4 / 1871526519666190923163010$

[10] Maurin M. [Brucellosis at the dawn of the 21st century]. Med Mal Infect. 2005; 35:6-16. [in French, English abstract]

[11] Mailles A, Garin-Bastuji B, Lavigne JP, Jay M, Sotto A, Maurin M, et al. Human brucellosis in France in the 21st century: results from national surveillance 2004-2013. Med Mal Infect. 2016; 46: 411-8. [in English, French title and abstract]

[12] Esmaeili H. Brucellosis in Islamic republic of Iran. J Med Bacteriol. 2015; 3:47-57.

[13] Mirnejad R, Jazi FM, Mostafaei S, Sedighi M. Epidemiology of brucellosis in Iran: a comprehensive systematic review and meta-analysis study. Microb Pathog. 2017; 109:239-47.

[14] Ghadimi-Moghadam A, Salahi M, Ghatee MA, GhadimiMoghadam A, Kanannejad Z, Mosav A, et al. Effect of human, livestock population, climatic and environmental factors on the distribution of brucellosis in southwest Iran. Acta Medica Mediterranea. 2020; 36:557-63.

[15] Jemal A, Clegg LX, Ward E, Ries LA, Wu X, Jamison PM, et al. Annual report to the nation on the status of cancer, 1975-2001, with a special feature regarding survival. Cancer. 2004; 101:3-27.

[16] Kim HJ, Fay MP, Feuer EJ, Midthune DN. Permutation tests for joinpoint regression with applications to cancer rates. Stat Med 2000; 19:335-51. (correction: 2001; 20:655).

[17] Nematollahi S, Ayubi E, Karami M, Khazaei S, Shojaeian M, Zamani $\mathrm{R}$, et al. Epidemiological characteristics of human brucellosis in Hamadan Province during 2009-2015: results from the National Notifiable Diseases Surveillance System. Int J Infect Dis. 2017; 61:56-61.

[18] Assafi M, Allu M, Abdulrahman I, Al-Berfkani M. The seroprevalence of human brucellosis in different age groups patients and other associated risk factors in Duhok, Iraq. Innovaciencia. 2019; 7:1-7.

[19] Aloufi AD, Memish ZA, Assiri AM, McNabb SJN. Trends of reported human cases of brucellosis, Kingdom of Saudi Arabia, 2004-2012. J Epidemiol Glob Health. 2016; 6:11-18.

[20] Makita K, Fèvre EM, Waiswa C, Kaboyo W, Eisler MC, Welburn SC. Spatial epidemiology of hospital-diagnosed brucellosis in Kampala, Uganda. Int J Health Geogr. 2011; 10:52. doi: 10.1186/1476072X-10-52

[21] Thakur SD, Kumar R, Thapliyal DC. Human brucellosis: review of an under-diagnosed animal transmitted disease. J Commun Dis. 2002; 34:287-301.

[22] Khazaei S, Shojaeian M, Zamani R, Mansori K, MohammadianHafshejani A, Rezaeian-Langroodi R, et al. Epidemiology and risk 
factors of childhood brucellosis in west of Iran. Int J Pediatr. 2016; 4: 2099-104

[23] Moosazadeh M, Abedi G, Kheradmand M, Safiri S, Nikaeen R. Seasonal pattern of brucellosis in Iran: a systematic review and meta-analysis. Iran J Health Sci 2016; 4:62-72.

[24] Kassiri H, Amani H, Lotfi M. Epidemiological, laboratory, diagnostic and public health aspects of human brucellosis in western Iran. Asian Pac J Trop Biomed. 2013; 3:589-94.

[25] Ageely H, Bani I, Gaffar A, Eltigani M, Yassin AO, Said B, Mahfouz MS. Prevalence and risk factors for brucellosis in Jazan Province, Saudi Arabia. Trop J Pharm Res. 2016; 15:189-94.

[26] Avdikou I, Maipa V, Alamanos Y. Epidemiology of human brucellosis in a defined area of Northwestern Greece. Epidemiol Infect. 2005; 133:905-10.
[27] Rostami H, Tavana AM, Tavakoli HR, Tutunchian M. Prevalence study of brucellosis in Iranian military forces during 2001-2009. J Health Pol Sustain Health. 2015; 1:191-94.

[28] Al-Tawfiq JA, Abukhamsin A. A 24-year study of the epidemiology of human brucellosis in a health-care system in Eastern Saudi Arabia. J Infect Pub Health. 2009; 2:81-5.

[29] Bokaie S, Sharifi L, Alizadeh H. Epidemiological survey of brucellosis in human and animals in Birjand, east of Iran. J Anim Vet Adv. 2008; 7:460-3.

[30] Kansiime C, Rutebemberwa E, Asiimwe BB, Makumbi F, Bazira J, Mugisha A. Annual trends of human brucellosis in pastoralist communities of south-western Uganda: a retrospective ten-year study. Infect Dis Poverty. 2015; 4:39. doi: 10.1186/s40249015-0072-y 